\title{
In-vitro models of human endometriosis (Review)
}

\author{
HONGJIE FAN \\ The Obstetrics and Gynecology Hospital of Fudan University, Shanghai 200011, P.R. China
}

Received July 2, 2019; Accepted December 6, 2019

DOI: $10.3892 /$ etm.2019.8363

\begin{abstract}
Endometriosis is one of the most common benign gynecological diseases in women of reproductive age worldwide. In past decades, a number of in-vitro models have been used to investigate the pathology and therapeutic methods for the treatment of endometriosis. The current review summarized the majority of currently available in-vitro models, which utilize a variety of cell or tissues types, including endometriotic cell lines, primary endometrial stromal cells, endometrial stem cells, endometrial explants, peritoneal explants and immune cells. These cells or tissues are cultured individually, co-cultured in $2 \mathrm{D}$ or 3D systems with various matrices or cultured in chicken chorioallantotic membranes and amniotic membranes culture systems. These models are able to represent one or more aspects of the process of endometriosis. These models are helpful and can be used to investigate the development of endometriosis and the underlying mechanisms of this disorder in detail, and help investigators select appropriate models for their experiments. Recently, the new concept of endometriosis as a fibrotic condition will lead research to investigate the differentiation of myofibroblasts and the development of fibrosis in endometriotic lesions, which will increase the development of novel models that can be used to investigate endometriotic fibrosis.
\end{abstract}

\section{Contents}

1. Introduction

2. In-vitro models

3. Discussion

\section{Introduction}

Endometriosis is defined as the presence of endometrial tissue (glands and stroma) outside of the uterine cavity, and is one of

Correspondence to: Dr Hongjie Fan, The Obstetrics and Gynecology Hospital of Fudan University, 419 Fang Xie Road, Shanghai 200011, P.R. China

E-mail: fan083@163.com

Key words: endometriosis, in-vitro model, endometrial stromal cell, endometrial explant, fibrosis the most common benign gynecological diseases in women of reproductive age worldwide (1). The most common site of endometriosis is the pelvic peritoneum and ovaries (1). Clinical symptoms of endometriosis are non-specific and include chronic pelvic pain, dysmenorrhea, deep pain during or after the sexual intercourse, infertility and irregular menstruation (1). Treatment choices vary significantly according to the symptoms of each patient, and include analgesics, hormonal treatment and surgical management $(1,2)$. All of the current treatments aim to only alleviate pain, regulate the menstrual cycle and promote fertility, but not to cure the disease completely. Recurrence of endometriosis is very high, ranging from $21.5 \%$ within 2 years to $40-50 \%$ within 5 years (3). Although endometriosis appears to be widely recognized and well-treated, the etiology and pathophysiology of this disease is still largely unknown. One of the well-documented and accepted theories is the retrograde menstruation theory, which states that viable menstrual endometrial cells retrograde back through the fallopian tubes into the peritoneal cavity and implant onto the peritoneal surface or ovaries (4). Based on this theory, a number of in-vitro and in-vivo models have been established to investigate the pathology behind endometriosis and its pathology. In animal models, the rhesus monkeys can develop endometriosis spontaneously, and endometriosis can be induced by transplanting endometrial tissues or cells from the same species or from humans into the peritoneum of primate or rodents, which has been reviewed in detail previously $(5,6)$. Animal models are able to recapitulate the potential pathogenesis of human endometriosis in vivo. However, they use different species other than humans, lack cellular and molecular investigations and require a large amount of capital and time. In-vitro models are easier to establish, can aid in understanding the process of endometriosis and can be used to investigate cellular and molecular mechanisms in detail. In the current review, the majority of in-vitro models to date are summarized and discussed, and further insights are obtained for the study of endometriosis.

\section{In-vitro models}

Cell lines. There are several endometriotic cell lines that are commercially available, including epithelial type $12-\mathrm{Z}, 49-\mathrm{Z}$, $108-\mathrm{Z}$ and $11-\mathrm{Z}$, and stromal type 22-B (7), which are immortalized endometriotic cells. By comparing these cells with primary human normal endometrial cells, it has been indicated that they exhibit a higher potency of cell migration and invasion, have a higher expression of genes regulating steroid 
metabolism, especially the hormone receptors (estrogen receptor $\beta$ and progesterone receptor), and produce a larger amount of prostaglandin (PG) E2, invasion related molecules [including the activity of matrix metalloproteinase (MMP)2 and MMP9], vascular endothelial growth factor (VEGF) and epidermal growth factor and cytokines [interleukin (IL)-1 $\beta$ and tumor necrosis factor (TNF) $\alpha$ ] (7). Similar to the immortalized normal endometrial cells, endometriotic cell lines also express genes related to hormone biosynthesis and signaling, cell cycle regulation, cell growth/survival and angiogenesis (7). These cell lines are widely used in vitro to investigate the mechanisms of action behind endometriosis due to them being a stable resource and the fact they are easy to handle.

In a cell monolayer culture model, the association between the function of endometriotic cells and hormonal related molecules has been studied. In this aforementioned study, cell proliferation and viability of $12-\mathrm{Z}$ and $22-\mathrm{B}$ cells was significantly decreased following transfection of siRNA designed to knock down the expression levels of EP2 and EP4 (receptors of PGE2; Fig. 1A) (8). In an endometriosis mouse model, an antagonist of growth hormone-releasing hormone, MIA-602, was demonstrated to significantly reduce the size of the endometriotic lesion (9). To investigate the underlying mechanisms of action, cell lines (12-Z and 49-Z) were exposed to MIA-602 in vitro (Fig. 1A). It was demonstrated that MIA-602 inhibited cell proliferation, reduced the expression of epithelial growth factor receptors and inhibited the activation of the MAP-kinases, ERK-1/2 (9). MicroRNA (miR)-145 was abundant in the ectopic endometrium, and the overexpression of miR-145 in 12-Z cells inhibited cell proliferation and invasion of the Matrige ${ }^{\circledR}$-coated invasion chamber (Fig. 1B). miR-145 was also indicated to reduce the expression of a number of cytoskeletal elements and stemness-associated factors in 12-Z cells (10). These results demonstrated that cell line models are beneficial tools to verify the results from clinical discoveries and murine models, as well as to explore the underlying mechanisms of action. They may provide information on novel biomarkers and treatment strategies for use in endometriosis.

Since endometriosis is recognized as a chronic inflammatory disease, the immune response of endometriotic cells have been investigated in vitro. Although endometriotic cell lines are not immune cells, they can respond to many immune regulators by producing cytokines or regulating cell functions, which indicate the importance of the immune response in the process of endometriosis and the close involvement of endometriotic cells in this inflammatory reaction (11). For example, IL-33 stimulates the production of pro-inflammatory cytokines in $12-\mathrm{Z}$ cells, including chemokine ligand (CXCL)1, IL-6, granulocyte-macrophage colony-stimulating factor and IL-15 (12) (Fig. 1A). CXCL12 and its specific receptor, chemokine receptor (CXCR)4 are highly expressed in patients with endometriosis, and the invasive and migratory ability of $12-\mathrm{Z}$ cells was increased by CXCL12 treatments, while this could be reversed by AMD3100, a CXCR4-specific inhibitor (13) (Fig. 1B).

In addition to the traditional 2D monolayer cultures, 3D models have also been established to mimic endometriosis using the endometriotic cell lines, EEC15 and 12-Z (Fig. 1C). In a previous study, cells were cultured in $1.5 \%$ polyHEMA-coated culture plates, and began to aggregate and form symmetrical spheroid structures within 7 days, which were visualized and measured under the microscope (14). The histological and molecular features of these 3D spheroids were similar to primary human endometriotic lesions. In these 3D models, molecules related to the immune response (including IL6, IL8, CXCL12 and CXCR4), micro-environmental interactions [(including MMP2 and hepatocyte growth factor (HGF)) and hormonal signaling [including prostaglandin-endoperoxide synthase 2 (PTGS2) and cytochrome P450 family 19 subfamily A member 1 (CYP19A1)] were significantly upregulated compared with the 2D models (14). Therefore, careful interpretation is required of the results from the $2 \mathrm{D}$ and $3 \mathrm{D}$ models using these cell lines, and 3D models using primary endometriotic cells and in vivo models are required to support the results gained.

Human primary endometrial epithelial cells (EECs) and endometrial stromal cells (ESCs). Compared with cell lines, human primary cells are a better model to use, with greater homology to the real in vivo situation. Human EECs and ESCs can be isolated from the eutopic endometrium and the ectopic endometriotic tissues, which allows for the maintenance of their individual in vivo phenotypic and functional markers (15). Isolated EECs and ESCs from deep endometriotic tissue have previously been immortalized using retroviral transfection of the human telomerase reverse transcriptase to develop individual cell lines, which maintain the characteristics of the primary cells, including the morphology and expression of hormone receptors (16). Estradiol induces the proliferation and invasion of ESCs from the eutopic endometrium of endometriosis through the phosphorylation of cofilin1 mediated by LIM domain kinase (LIMK)1, which indicated that the LIMK1/cofilin1 pathway may be a therapeutic target for this disease (17). Celecoxib is a selective cyclooxygenase (COX)-2 inhibitor. In EECs isolated from the eutopic endometrium of endometriosis patients, Celecoxib inhibited the proliferation, induced the apoptosis and reduced the secretion of PGE2 and VEGF (Fig. 1A) (18). To investigate the association between the extracellular matrix stiffness and the behavior of the endometriotic stromal cells, ESCs are grown on top of polyacrylamide gels with a variety matrix stiffness (Fig. 1C). The increased matrix stiffness induces the formation of F-actin stress fibers and the expression of procollagen I in cells from healthy patients and patients with endometriosis, while the production of alpha smooth muscle actin ( $\alpha$ SMA)-containing stress fibers was only induced in the endometriotic stromal cells. The increased matrix stiffness also regulated the expression of molecules related to collagen synthesis and degradation (such as type I collagen, cyclin D1, MMP-1 and MMP-14), which suggests that an aberrant extracellular matrix is involved in the process of endometriosis (19).

Similar to cell lines, primary endometrial cells can be used to generate 3D models (Fig. 1D). The 3D polymerized collagen matrices contain the endometriotic or ESCs mixed with the type I collagen solution. After polymerization, the proliferation of the ESCs in the 2D culture were significantly less inhibited compared with the ESCs in the 3D polymerized collagen, and the activation of AKT and ERK pathway was more intense in the endometriotic stromal cells than that in the ESCs. The inhibition of the AKT and ERK signaling pathway decreases 
A

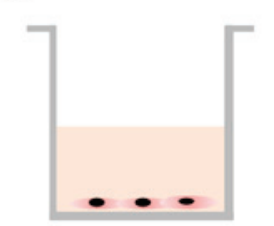

D

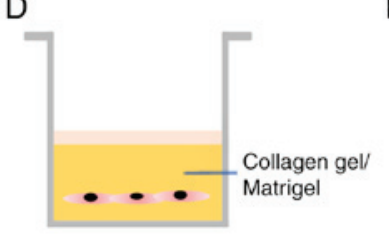

G

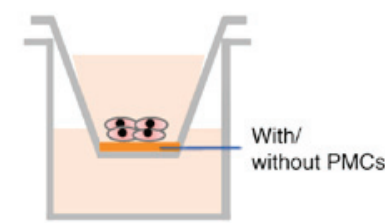

B

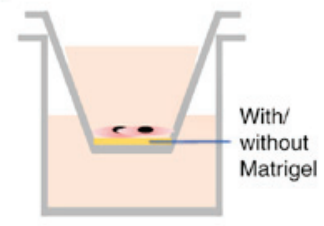

$\mathrm{E}$

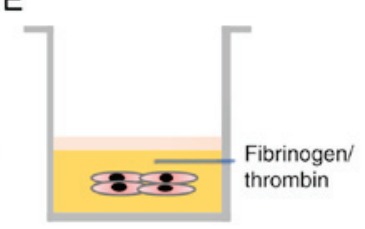

$\mathrm{H}$

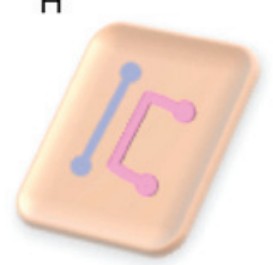

C

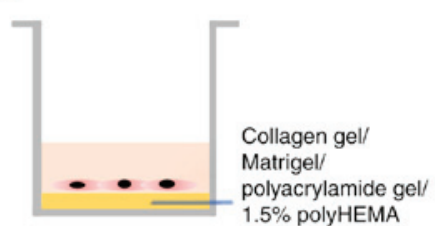

$\mathrm{F}$
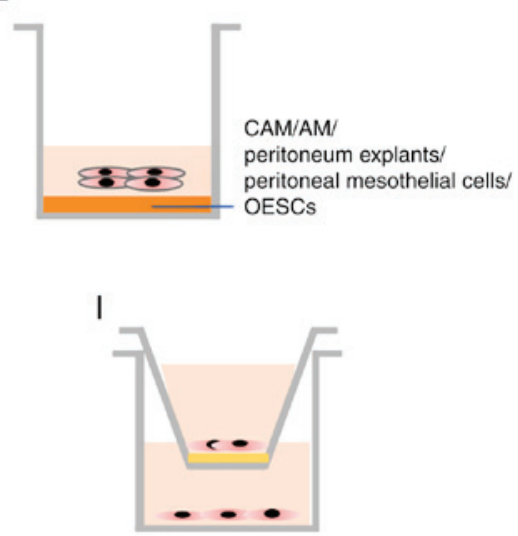

Figure 1. In vitro models of endometriosis. (A) Endometriotic cell lines, primary EECs and ESCs, EN-MSCs, OESCs and PMCs are cultured in plates. (B) The migration and invasion of the endometriotic cell lines, ESCs, and mixed population with ESCs and PMCs in a chamber with/without Matrigel. (C) Endometriotic cell lines, EEC and ESCs are grown on top of plates coated with various matrices. (D) 3D model of endometriotic cell lines, ESCs, EN-MSCs and OSE cells in collagen gel or Matrigel solution. (E) 3D model of endometrial explants in fibrinogen or thrombin solution. (F) Endometrial explants and cell lines are grown on top of CAM, AM, peritoneum explants, PMCs or OESCs. (G) Endometrial explants or ESCs grown in inserts coated with/without PMCs. (H) The microfluidic channels model with ESCs and PMCs. (I) EECs, ESCs, EN-MSCs, PMCs, immune cells and human umbilical cord endothelial cells are cultured in inserts or in the lower well in various combinations. 3D, three dimensional; AM, amniotic membrane; CAM, chicken chorioallantotic membrane; EEC, endometrial epithelial cells; EN-MSC, endometrial mesenchymal stem cells; OESC, ovarian endometrioma stromal cells; ESC, endometrioma stromal cells; PMC, peritoneal mesothelial cells.

the cell proliferation but does not increase the caspase 3/7 activity in the $3 \mathrm{D}$ system of the endometriotic stromal cells (20). These results demonstrated that differences exist between the endometrial cells from patients with endometriosis and that from women without endometriosis. By further comparing these two cell types, it is likely that more knowledge can be obtained about the pathology behind endometriosis. Similar to cell lines, primary cells still perform differently in 2D and 3D culture systems, which researchers should take account of when designing experiments and interpreting results.

Previous results have indicated that the peritoneal fluid (PF) may be associated with the process of the endometriosis. PF collected from patients with endometriosis or women without endometriosis exhibit a similar effect on the cell proliferation of the eutopic EECs and ESCs in vitro (Fig. 1A) (21), and induce the expression of VEGF-A, urokinase plasminogen activator and plasminogen activator inhibitor-1 in the eutopic ESCs. $\mathrm{PF}$ from patients with endometriosis reduces the expression of a number of miRs in the eutopic ESCs compared with the PF from women without endometriosis, including miR-16-5p, miR-29c-3p and miR-424-5p (22,23). Overexpression of these miRs reduces the secretion of VEGF-A in eutopic ESCs $(22,23)$. These results demonstrated that PF contributes to the endometriosis process, especially during angiogenesis, via the regulation of miRs.

Using the primary cell model, factors regulating endometriosis have been previously investigated, including platelet-derived growth factor (24), which is a macrophage secretory product, and miR-200c and miR-145 (10,25), which regulate the proliferation of eutopic ESCs. Similarly, the anti-fibrotic factor epigallocatechin-3-gallate (EGCG), has been revealed to inhibit the proliferation, migration and invasion of eutopic and ectopic ESCs (26). EGCG also significantly decreases the expression levels of $\alpha$ SMA, Collagen-I (Col-I), connective tissue growth factor (CTGF) and fibronectin (FN) in both cell types (26). Di-(2-ethylhexyl)-phthalate, which is an environmental contaminant, induces the invasion of the eutopic ESCs through the activation of MMP-2 and 9 and the ERK signaling pathway (27). Inhibition of the Wnt- $\beta$-catenin pathway significantly decreases the expression of the fibrotic marker genes $\alpha \mathrm{SMA}$, Col-I, CTGF and FN in endometriotic and ESCs, and activation of the Wnt- $\beta$-catenin pathway not only induces the expression of these four markers, but also increases the cell proliferation and migration of ESCs (28). By analyzing the secretion in the culture medium of ESCs and EECs, a number of factors associated with angiogenesis have been detected $(29,30)$, including platelet-derived endothelial cell growth factor, VEGF, macrophage migration inhibitory factor and IL-8.

The aforementioned in-vitro models were based on monocultures of EECs or ESCs. Co-culturing ESCs and ECCs may represent the whole endometrium (Fig. 1A). In this model, IL-8 increases cell survival with no differences observed between cells from women with or without endometriosis, while IL-12 inhibits cell survival from women without endometriosis, but not in cells obtained from women with endometriosis (31). When these endometrial cells are grown on top of the solidified 
Matrigel solution (Fig. 1C), the micro-tubular structures are visualized under the microscope as a simulation of angiogenesis. Overexpression of protein tyrosine phosphatase (PTEN) increases cell apoptosis, inhibits the cell cycle and inhibits the formation of the micro-tubular structures, with the reciprocal observations indicated when PTEN is inhibited (32). However, in this model, it was impossible to differentiate the effect of ILs and PTEN on EECs or ESCs. The ratio of EECs and ESCs from individual patients was not consistent throughout the aforementioned study, and the reproducibility of the results is a concern.

Endometrial stem cells. The endometrial stem cell implantation theory is an expansion of the retrograde menstruation theory (33). Endometrial epithelial progenitor cells and mesenchymal stem-cell-like cells, together with the menstruation are shed into the peritoneum, where they implant and establish the ectopic lesions. Cells isolated from the ectopic endometrium (endometriosis lesions) express markers of multipotent mesenchymal stem cells (MMSCs), including CD90, CD73 and CD105, and these cells are capable of adipogenic and osteogenic differentiation. Although differences are indicated between the MMSCs from the eutopic endometrium and from the ectopic endometrium, the similarities suggest that the ectopic MMSCs can be used as a novel model for investigating endometriosis $(34,35)$. Endometrial mesenchymal stem cells (EN-MSCs) are isolated from uterine and ovarian endometriotic tissues from the same patient as eutopic EN-MSCs and ectopic EN-MSCs. The two cells have different gene profiles, especially IL-1b and COX-2, which are more highly expressed in the ectopic EN-MSCs, and enhance the migratory and invasive ability of ectopic EN-MSCs (36). The EN-MSCs collected from the eutopic and ectopic endometrial tissues represent a heterogenic population of mesenchymal stem cells and stromal fibroblast cells. When the EN-MSCs are directly co-cultured with the endothelial cell line, human umbilical vein endothelial cells (HUVECs), EN-MSCs differentiate into endothelial cells and the co-cultures form tube-like structures in Matrigel (Fig. 1C) (37), which can be used as a model for studying the angiogenesis of endometriosis in vitro.

Recently, Hapangama et al (38) identified endometrial basalis-like (SSEA1+/SOX9+) cells contributing to the pathogenesis of endometriosis. The basalis-like cells (SSEA1+, SOX9+) were abundant in the functional layer during the secretory phase, from the eutopic endometrium of patients with endometriosis. In-vitro differentiation experiments demonstrated that SSEA1 + EECs are not pluripotent as they are unable to differentiate down the same mesodermal lineages as EN-MSCs. However, the cells from patients with endometriosis are able to generate ectopic endometriotic lesion-like structures in a 3D model with Matrigel (Fig. 1D), and these structures are morphologically similar to the ectopic endometrial epithelium. This 3D model expresses cytokeratin18, MUC-1, $\beta$-catenin and the hormone receptors (ER $\alpha, E R \beta$ and PR). The evidence indicated the importance of SSEA1+/SOX9+ cells in the development of endometriosis, which may be a potential therapeutic target for endometriosis.

Endometrial explants. Along with isolated endometrial cells, endometrial explants could also be utilized for the establishment of models to investigate endometriosis. Small endometrial fragments could outgrow, form glands/vessels, proliferate and invade the $3 \mathrm{D}$ culture models with a fibrinogen/thrombin solution, and could be used to study the early events of endometriosis $(39,40)$ (Fig. 1E). The tubular structures in 3D models have been examined using transmission electron microscopy, and these cells are polarized, with microvilli on the apical surface and present as desmosome-like structures on the basement membrane, with features that are consistent with the glandular epithelial cells observed in vivo (41). In this model, treatment with dienogest, which is a current treatment for endometriosis, inhibits the outgrowth of endometrial explants and induces apoptosis (41). A total of two angiogenic factors, glycodelin and COX-2 have been detected in $>80 \%$ of $3 \mathrm{D}$ cultures, which indicates that angiogenesis occurs in this model (42). Statin inhibits cell proliferation and impairs angiogenesis in a concentration-dependent manner, which implies that statin may be a potential treatment strategy for the early stages of endometriosis (43). In this 3D model, only the normal endometrium was used, but endometriotic endometrium should be used to investigate the pathogenesis of the disorder and for drug screening. The endometrial explants took a number of weeks to generate a fully developed model, and an obvious disadvantage to this model is that it is time-consuming compared to cell lines or primary endometrial cells. However, the glandular formation and angiogenesis are great merits of this 3D model.

Chicken chorioallantoic membranes (CAMs) and amniotic membranes culture model. In the retrograde menstruation theory, endometrium encounters the peritoneum, implants, invades and forms the ectopic endometrium and endometriotic lesions (33). Therefore, the peritoneum represents the other side of the endometriosis pathogenesis. In vitro, selected biological membranes have successfully replaced the peritoneum, such as the CAM, which can be attached to and be invaded by the endometrium (Fig. 1F). The CAM assay is widely used to investigate the angiogenic properties of the endometrium (44). Only endometrial fragments, instead of single endometrial cells, have been demonstrated to induce the formation of endometriosis-like lesions within $72 \mathrm{~h}$ after implanting onto the CAM (45). A previous study demonstrated that the endometrium collected from women, who were using oral contraceptives (OCs), presented fewer endometriosis-like lesions in the CAM compared with the endometrium collected from the menstrual endometrium from women experiencing normal cycles (46), which suggested that OCs affected the characteristics of the endometrium and aided in the regulation of endometriosis. In this model, the angiostatic compounds, such as the anti-hVEGF antibody, TNP-470, endostatin and anginex, impair the formation of endometriosis-like lesions and decrease vessel densities. This suggests that the angiostatic compounds may provide an alternative therapy for endometriosis (47).

Similar to the CAM, the human amniotic membranes (AM) can also be used as a surrogate for the peritoneum $(48,49)$. Endometrial fragments are unable to adhere to the intact epithelial side of the AM; however, they can adhere to the damaged and non-epithelial side. Endometrial carcinoma cell lines have been indicated to attach to both sides of the AM, which may suggest that primary endometrial fragments are superior to endometrial cell lines in this model. This also 
indicates that the integrity of the epithelia on the peritoneum may prevent the adhesion and growth of the retrograde endometrium, prohibiting the initial stages of endometriosis $(50,51)$ (Fig. 1F). When AMs are stripped of the epithelial lining, the endometrial fragments, but not the isolated primary endometrial cells, can adhere to both sides of the membrane $(48,49)$. The isolated endometrial cells are associated with lower expression levels of $\beta 1$ integrins as well as E- and P-cadherin.

The CAMs and AMs are good models to use in the study of the endometrium implantation, the formation of endometriotic lesion and the angiogenesis of endometrium in vitro. These models have stable resources, a simplified procedure and a standard analysis of results $(44,49)$.

Co-culture models with peritoneal cells and explants. In addition to CAMs and AMs, human primary peritoneum tissues are also used to create in vitro co-cultures with endometrium (Fig. 1F). The peritoneum explants collected from the anterior abdominal wall are cut into small pieces and cultured in plates or inserts, and endometrium biopsies are added to the top of peritoneum explants. Within 2 days, endometrial cells attach and invade the tissue $(48,52,53)$. The majority of endometrial fragments attach to a peritoneum denuded of mesothelial cells $(48,54)$. However, when mesothelial cells are isolated from the PF from normal women and cultured to form a monolayer in the collagen-coated plates, the endometrial fragments can also grow on top of the monolayer, demonstrating the characteristic morphology of endometriosis in vivo (Fig. 1F) (55). ESCs are also able to attach to the peritoneum mesothelial cell (PMC) monolayer (56). In adhesion assays, the labelled endometrial epithelial carcinoma cell line, CRL-1671, attaches to the human mesothelial cell line, CRL-9444, and pretreatment of mesothelial cells with the cytokines TNF $\alpha$, IL-6 and IL-8, inhibits the number of attached EECs (57). In the same model, ESCs attach and invade through the LP-9 PMC monolayer, and the treatment of ESCs with an active tyrosine kinase inhibitor, Imatinib, does not affect the attachment of ESCs onto the LP-9 monolayer, but does inhibit the invasion of the ESCs (58). In order to investigate the role of PMCs in the invasion of ESCs, Nair et al (59) developed a model where ESCs invaded through the Matrigel-coated chamber with or without the presence of PMCs growing on top of the Matrigel (Fig. 1G). In this aforementioned study, it was indicated that with the presence of the PMCs monolayer, the invasive ability of the ESCs was increased, and the transcription of extracellular signal-related kinase, colony stimulating factor-1, c-fms and c-Met were also increased in ESCs and PMCs after the endometrial-PMC attachment (59). This demonstrated that PMCs contributed to endometrial invasion.

Chen et al (60) established a novel model using microfluidic channels system to observe the interaction of PMCs and ESCs in real time (Fig. 1H). In this model, two microfluidic channels were designed in a glass slide, and PMCs and ESCs were incubated in the two channels, respectively. After removal of the channel mold, these two cell types migrate and interact with each other. On day 3 of culture, the two cell types contact, and the invasion and interaction of these cells can be visualized on day 7. PMCs from control patients were indicated to resist the invasion of ESCs, irrespective of whether the cells were from patients with or without endometriosis, but PMCs from patients with endometriosis were able to be invaded by normal and endometriotic ESCs (60). The endometriotic PMCs lose their adhesion and undergo apoptosis when invaded by ESCs. These results indicated the critical role of intact PMCs in the pathogenesis of endometriosis.

Using peritoneal cells or explants together with endometrium tissue, instead of surrogates, to establish in vitro models is a step closer to mimicking the real in vivo environment. This approach reconstructs the relationship between the ectopic endometrium and the peritoneum. The disadvantage of these models is the diversity of cell types, the unstable cell or tissue resources and the complexity to its formation, which makes it difficult to reproduce these experiments in respective labs.

Co-culture models with immune cells. It is well-known that the immune system is associated with the development of endometriosis, including macrophages and a number of cytokines (11). In addition to the culture system with secretory immune-factors, endometrium co-cultures with immune cells can be used to study the immune reaction observed in endometriosis. A previous study used U937 (human immune cells monocyte cell line), HMrSV5 (human PMC cell line) and eutopic ESCs from patients with endometriosis to generate a number of co-culture models with a variety of combinations of these three types of cells, including as a direct co-culture of any two combinations of the cells with or without the third cell type, cultured in the transwell chamber inserts and a directly co-culture of all three cell types (Fig. 1I). It was indicated that co-cultures of the three cell types promoted the secretion of RANTES, a chemoattractant for both monocytes and activated T-cells, as well as macrophage-inflammatory protein-1 $\alpha$ (MIP-1 $\alpha$ ) compared to individual cell cultures of these three cell types and co-culturing of any two types of cells (61). The combination of 17b-estradiol and dioxin 2,3,7,8-tetrachlorodibenzo-p-dioxin (TCDD; an environmental contaminant) increases the secretion of RANTES and MIP- $1 \alpha$ in these models, promotes the invasiveness of ESCs and increases the expression of MMP-2 and MMP-9 in ESC, which is mediated by CC-motif chemokines (61). These observations may indicate that $17 \mathrm{~b}$-estradiol and TCDD promote the onset of endometriosis (61). In another model, macrophages from the PF of patients with endometriosis or control women have been cultured indirectly with EECs or ESCs in the lower well of transwell chambers (Fig. 1I). PF macrophages from endometriosis increase the proliferation of EECs and ESCs compared with control women (62), which suggests that macrophages from patients with endometriosis have developed differences compared with those from women without endometriosis. Further investigation is required to identify these differences and to elicit the mechanism of action in endometriosis.

Ovarian endometriosis model. As well as the peritoneum, ovaries are another common ectopic site that the retrograde endometrium is able to adhere to and grow (33). Ovarian endometrioma stromal cells (OESCs) can be used to establish in vitro endometriosis models (Fig. 1A). Daidzein-rich isoflavone aglycones, which is a dietary supplement, inhibits the proliferation of OESCs and reduces the expression of IL-6, IL-8, COX-2 and aromatase in OESCs (63). To study the association of miR-214 and fibrosis in endometriosis, the overexpression of miR-214 can be used, and in OESCs, significantly decreases the mRNA expression of $\alpha$ SMA, Col-I and CTGF (64). EEC16 cells are an 
Table I. Functions of in vitro models of endometriosis.

A, Proliferation

\begin{tabular}{lc}
\hline Models & (Refs) \\
\hline Cell lines & $8-10$ \\
Primary EECs, ESCs & $10,18,21,25,26$ \\
OESCs & 63 \\
Primary EECs, ESCs in 3D & 20 \\
Stem cells & 36 \\
Immune cell cultured with ESCs & 62 \\
\hline
\end{tabular}

B, Migration, invasion

\begin{tabular}{lc}
\hline Models & (Refs) \\
\hline Migration, invasion Cell lines & 10,13 \\
Primary EECs, ESCs & $16,26,28$ \\
Stem cells & 36 \\
Endometrial fragments and peritoneum & $48,52,53$ \\
ESCs and peritoneal cell monolayer & 57,58 \\
Microfluidic channels system with PMCs & 60 \\
and ESCs & \\
Immune cell cultured with ESCs & 61 \\
\hline
\end{tabular}

C, Inflammatory response

\begin{tabular}{lc}
\hline Models & (Refs) \\
\hline Cell lines & 12,13 \\
OESCs & 63 \\
Cell lines in 3D & 14 \\
Immune cell cultured with ESCs & 11,61 \\
\hline
\end{tabular}

D, Formation of endometriotic lesions or glands

Models

(Refs)

Cell lines in 3D

Stem cell in 3D

Endometrial explants in 3D

39,40

Endometrial explants in CAM

Endometrial explants and mesothelial

cells

OSEs and ESCs in 3D

66

E, Extracellular matrix regulation and fibrosis

\begin{tabular}{lc}
\hline Models & (Refs) \\
\hline Cell lines & 10 \\
Primary EECs, ESCs & $19,22,23,26,27,28$ \\
OESCs & 64,68 \\
\hline
\end{tabular}

Table I. Continued. Functions of in vitro models of endometriosis.

F, Angiogenesis

\begin{tabular}{lc}
\hline Models & (Refs) \\
\hline Primary EECs, ESCs & 29,30 \\
EECs and ESCs in matrigel & 32 \\
EN-MSCs and HUVECs & 37 \\
Endometrial explants in 3D & 42,43 \\
Endometrial explants in CAM & 47 \\
\hline G, Implantation & (Refs) \\
\hline Models & 45 \\
\hline $\begin{array}{l}\text { Endometrial explants in CAM } \\
\text { Endometrial explants in AM }\end{array}$ & $48-51$ \\
ESCs and PMCs & 56 \\
\hline EECs, endometrial epithelial cells; ESCs, endometrial stromal cells; \\
$\begin{array}{l}\text { OESCs, stromal cells from ovarian endometrioma; PMCs, perito- } \\
\text { neum mesothelial cells; CAM, chicken chorioallantoic membrane; } \\
\text { AM, amniotic membrane; OSEs, ovarian surface epithelial cells; } \\
\text { EN-MSCs, endometrial mesenchymal stem cells. }\end{array}$
\end{tabular}

ovarian endometriosis epithelial cell line that are isolated from superficial endometriosis lesions on the surface of the ovaries, which express a number of biomarkers at different levels when compared to normal ovarian epithelial cells, including the lack of N-Cadherin expression (14). EEC16 can form smooth spheroids in $1.5 \%$ polyHEMA-coated culture plates to generate a $3 \mathrm{D}$ model which mimics endometriotic lesions (Fig. 1C). In this model, a number of genes associated with immune responses, micro-environmental interactions and hormonal signaling were upregulated, including IL6, IL8, MMP2, HGF, PTGS2 and CYP19A1 (14). EEC16 cells can be immortalized using TERT, but EEC16-TERT cells differentially express epithelial and mesenchymal markers, specifically, they do not express cytokeratin nor other epithelial markers, including BerEP4, but do express vimentin. EEC16 cells express keratin and vimentin but lack E-cadherin expression (65). The colony formation ability of the EEC16-TERT cell line has been indicated to be inhibited by the Src and Wnt signaling pathways, respectively, which may provide a novel strategy to manage endometriosis (65).

Human ovarian surface epithelial (OSE) cells from healthy ovaries together with ESCs and 17 $\beta$-estradiol (E2) form a lumen structure in the collagen gel (Fig. 1D), while OSEs alone can only form circular arrangements. Without E2, no lumen structure is detected. In this 3D model, OSE cells form a lumen structure surrounded by ESCs. Cytokeratin and epithelial membrane antigen were detected in glandular cells, and cilia were observed on the cell surface (66), which revealed that there was an interaction between ESCs and ovarian cells, and an importance of E2 in the process of endometriosis. The disadvantage of this model with the collagen gel solution is that the 
gel shrinks within 3 days, is released from the culture well and floats in the medium. The gel divides into multiple small pieces after 9 to 12 days. Therefore, it is important to define the exact time point to observe the lumen structure, making observations difficult and meaning experienced researchers are required.

\section{Discussion}

Endometriosis is a complex disease involving a number of processes, including implantation, proliferation, invasion, angiogenesis and apoptosis resistance (33). A variety of signaling pathways are involved in the development and progression of this disease. Various in vitro models have been established to study the pathogenesis of endometriosis in the past decades, and the molecular mechanisms of action and potential therapies for this disease have also been widely investigated $(7,15,21,33,38-40,44,50,60,61)$. Each model exhibits unique characteristics and functions, and can represent one or a number of aspects of endometriosis as summarized in Table I, including cell proliferation, invasion, angiogenesis and formation of endometriotic lesions. Cell lines and primary cells can be used to study the majority of aspects of the endometriosis process, except the formation of endometriotic lesions, which requires a $3 \mathrm{D}$ matrix to provide sufficient solid space. For angiogenesis, co-culturing with CAM/AM or HUVECs is preferable than culturing primary cells or tissues only. It should be noted that cell lines and primary cells in different situations, including in 2D or 3D models, demonstrate distinct potencies in regards to proliferation and the production of factors related to the immune response and hormonal signaling $(14,20)$. Based on the purpose of the study and the available resources of cells or tissues, investigators should select appropriate models or establish a novel model of their own.

When using cell lines to study the process of endometriosis, results should be carefully interpreted due to their carcinomic origins varying from that of human primary endometriotic cells. Alternatively, human primary EECc and ESCs are ideal cells to generate models, especially in 3D models with peritoneum cells and explants, which provide a reliable situation to investigate cell-cell interactions, cross-talks and angiogenesis. These models emulate the in vivo processes, aid in the clarification of the potential mechanisms of action and can be used to identify therapeutic advances $(20,31)$. By co-culturing endometriotic cells with immune cells, the mechanisms of action behind the inflammatory process in endometriosis can be further investigated. Each model has only demonstrated one or several aspects of endometriosis, but not the whole picture. Currently, a number of representative models of endometriosis have been generated in various laboratories, with no consensus of opinions on the standardization of models being achieved. More comprehensive and reliable in vitro models are required to establish increasingly detailed studies.

Vigano et al (67) proposed a new concept of the definition of endometriosis in 2017, according to its pro-fibrotic nature. It has been demonstrated that the endometrial stroma and glands are only present as a minor component of endometriotic lesions, and are often absent in some forms of the disease, including rectovaginal nodules and ovarian endometrioma. Additionally, alterations in smooth muscle and fibrosis are consistent features of all forms of the disease (67). Therefore, the definition of endometriosis needs to be reconsidered and reworded as 'a fibrotic condition in which endometrial stroma and epithelium can be identified'. The fibrosis is a condition caused by the accumulation and contraction of the collagenous extracellular matrix, which is produced by activated myofibroblasts (67). Therefore, the differentiation and activation of myofibroblasts in endometriotic lesions should be a focus of future research. Currently, the model for studying myofibroblasts is the differentiation of ESCs. For example, endometriotic stromal cells isolated from the ovarian endometrioma are driven into the epithelial-mesenchymal transition and the fibroblast-to-myofibroblast trans-differentiation by activated platelets through the TGF- $\beta /$ Smad signaling pathway, which results in increased cell contractility, collagen production and ultimately to the fibrosis (68). In the future, 3D culture or co-cultures with peritoneal explants and immune cells could also be used to investigate the fibrosis and its dynamic changes, as well as its interaction with the peritoneum and the immune system. Except for understanding the development and mechanisms of action behind fibrosis in endometriosis, in vitro models are a promising tool to investigate therapeutic advances for managing endometriosis.

\section{Acknowledgements}

Not applicable.

\section{Funding}

No funding was received.

\section{Availability of data and materials}

Not applicable.

\section{Authors' contributions}

HF designed and wrote the manuscript.

\section{Ethics approval and consent to participate}

Not applicable.

\section{Patient consent for publication}

Not applicable.

\section{Competing interests}

The author declare that they have no competing interests.

\section{References}

1. Kuznetsov L, Dworzynski K, Davies M and Overton C; Guideline Committee: Diagnosis and management of endometriosis: Summary of NICE guidance. BMJ 358: j3935, 2017.

2. Dunselman GA, Vermeulen N, Becker C, Calhaz-Jorge C, D'Hooghe T, De Bie B, Heikinheimo O, Horne AW, Kiesel L, Nap A, et al: ESHRE guideline: Management of women with endometriosis. Hum Reprod 29: 400-412, 2014.

3. Guo SW: Recurrence of endometriosis and its control. Hum Reprod Update 15: 441-461, 2009. 
4. Halme J, Hammond MG, Hulka JF, Raj SG and Talbert LM: Retrograde menstruation in healthy women and in patients with endometriosis. Obstet Gynecol 64: 151-154, 1984.

5. Bruner-Tran KL, Mokshagundam S, Herington JL, Ding T and Osteen KG: Rodent models of experimental endometriosis: Identifying mechanisms of disease and therapeutic targets. Curr Womens Health Rev 14: 173-188, 2018.

6. King CM, Barbara C, Prentice A, Brenton JD and Charnock-Jones DS: Models of endometriosis and their utility in studying progression to ovarian clear cell carcinoma. J Pathol 238: 185-196, 2016.

7. Banu SK, Lee J, Starzinski-Powitz A and Arosh JA: Gene expression profiles and functional characterization of human immortalized endometriotic epithelial and stromal cells. Fertil Steril 90: 972-987, 2008.

8. Lee J, Banu SK, Rodriguez R, Starzinski-Powitz A and Arosh JA Selective blockade of prostaglandin E2 receptors EP2 and EP4 signaling inhibits proliferation of human endometriotic epithelial cells and stromal cells through distinct cell cycle arrest. Fertil Steril 93: 2498-2506, 2010.

9. Köster F, Jin L, Shen Y, Schally AV, Cai RZ, Block NL, Hornung D, Marschner G, Rody A, Engel JB and Finas D: Effects of an antagonistic analog of growth hormone-releasing hormone on endometriosis in a mouse model and in vitro. Reprod Sci 24 1503-1511, 2017.

10. Adammek M, Greve B, Kassens N, Schneider C, Brüggemann K, Schüring AN, Starzinski-Powitz A, Kiesel L and Götte M: MicroRNA miR-145 inhibits proliferation, invasiveness, and stem cell phenotype of an in vitro endometriosis model by targeting multiple cytoskeletal elements and pluripotency factors. Fertil Steril 99: 1346-1355.e5, 2013.

11. Králíčková M Fiala L, Losan P, Tomes $P$ and Vetvicka V: Altered immunity in endometriosis: What came first? Immunol Invest 47: 569-582, 2018

12. Miller JE, Monsanto SP, Ahn SH, Khalaj K, Fazleabas AT, Young SL, Lessey BA, Koti M and Tayade C: Interleukin-33 modulates inflammation in endometriosis. Sci Rep 7: 17903, 2017.

13. Ruiz A, Ruiz L, Colòn-Caraballo M, Torres-Collazo BJ, Monteiro JB, Bayona M, Fazleabas AT and Flores I: Pharmacological blockage of the CXCR4-CXCL12 axis in endometriosis leads to contrasting effects in proliferation, migration, and invasion. Biol Reprod 98: 4-14, 2018.

14. Brueggmann D, Templeman C, Starzinski-Powitz A, Rao NP Gayther SA and Lawrenson K: Novel three-dimensional in vitro models of ovarian endometriosis. J Ovarian Res 7: 17, 2014.

15. Ryan IP, Schriock ED and Taylor RN: Isolation, characterization, and comparison of human endometrial and endometriosis cells in vitro. J Clin Endocrinol Metab 78: 642-649, 1994.

16. Boccellino M, Quagliuolo L, Verde A, La Porta R, Crispi S, Piccolo MT, Vitiello A, Baldi A and Signorile PG: In vitro model of stromal and epithelial immortalized endometriotic cells. J Cell Biochem 113: 1292-1301, 2012.

17. Liu J, Zhang Z, Liu J and Wang D: LIM Kinase 1 mediates estradiol effects on the phosphorylation of Cofilin1 in eutopic endometrial stromal cells during the invasion and proliferation of endometriosis. Reprod Sci 26: 1499-1505, 2019.

18. Olivares C, Bilotas M, Buquet R, Borghi M, Sueldo C, Tesone M and Meresman G: Effects of a selective cyclooxygenase-2 inhibitor on endometrial epithelial cells from patients with endometriosis. Hum Reprod 23: 2701-2708, 2008.

19. Matsuzaki S, Canis M, Pouly JL and Darcha C: Soft matrices inhibit cell proliferation and inactivate the fibrotic phenotype of deep endometriotic stromal cells in vitro. Hum Reprod 31: $541-553,2016$

20. Matsuzaki S and Darcha C: Co-operation between the AKT and ERK signaling pathways may support growth of deep endometriosis in a fibrotic microenvironment in vitro. Hum Reprod 30: 1606-1616, 2015

21. Overton CE, Fernandez-Shaw S, Hicks B, Barlow DH and Starkey P: In vitro culture of endometrial stromal and gland cells as a model for endometriosis: The effect of peritoneal fluid on proliferation. Fertil Steril 67: 51-56, 1997.

22. Braza-Boïls A, Salloum-Asfar S, Marí-Alexandre J, Arroyo AB, González-Conejero R, Barcelo-Mólina M, García-Oms J, Vicente V, Estelles A, Gilabert-Estelles J and Martínez C: Peritoneal fluid modifies the microRNA expression profile in endometrial and endometriotic cells from women with endometriosis. Hum Reprod 30: 2292-2302, 2015.
23. Braza-Boïls A, Gilabert-Estélles J, Ramon LA, Gilabert J, Marí-Alexandre J, Chirivella M, Espana F and Estelles A: Peritoneal fluid reduces angiogenesis-related microRNA expression in cell cultures of endometrial and endometriotic tissues from women with endometriosis. PLoS One 8: e62370, 2013.

24. Surrey ES and Halme J: Effect of platelet-derived growth factor on endometrial stromal cell proliferation in vitro: A model for endometriosis? Fertil Steril 56: 672-679, 1991.

25. Liang Z, Chen Y, Zhao Y, Xu C, Zhang A, Zhang Q, Wang D, He J, Hua W and Duan P: miR-200c suppresses endometriosis by targeting MALAT1 in vitro and in vivo. Stem Cell Res Ther 8: 251,2017

26. Matsuzaki S and Darcha C: Antifibrotic properties of epigallocatechin-3-gallate in endometriosis. Hum Reprod 29: 1677-1687, 2014.

27. Kim SH, Cho S, Ihm HJ, Oh YS, Heo SH, Chun S, Im H, Chae HD, Kim CH and Kang BM: Possible role of phthalate in the pathogenesis of endometriosis: In vitro, animal, and human data. J Clin Endocrinol Metab 100: E1502-E1511, 2015.

28. Matsuzaki $S$ and Darcha $C$ : Involvement of the Wnt $/ \beta$-catenin signaling pathway in the cellular and molecular mechanisms of fibrosis in endometriosis. PLoS One 8: e76808, 2013.

29. Laschke MW and Menger MD: In vitro and in vivo approaches to study angiogenesis in the pathophysiology and therapy of endometriosis. Hum Reprod Update 13: 331-342, 2007.

30. Fujimoto J, Sakaguchi H, Hirose R and Tamaya T: Expression of platelet-derived endothelial cell growth factor (PD-ECGF) related to angiogenesis in ovarian endometriosis. J Clin Endocrinol Metab 84: 359-362, 1999.

31. Gazvani R, Smith L and Fowler PA: Effect of interleukin-8 (IL-8), anti-IL-8, and IL-12 on endometrial cell survival in combined endometrial gland and stromal cell cultures derived from women with and without endometriosis. Fertil Steril 77: 62-67, 2002.

32. Lv J, Zhu Q, Jia X, Yu N and Li Q: In vitro and in vivo effects of tumor suppressor gene PTEN on endometriosis: An experimental study. Med Sci Monit 22: 3727-3736, 2016.

33. Wang Y, Nicholes K and Shih IM: The origin and pathogenesis of endometriosis. Annu Rev Pathol: Sep 3,2019 (Epubahead of print).

34. Savilova AM, Yushina MN, Rudimova YV, Khabas GN Chuprynin VD and Sukhikh GT: Characteristics of multipotent mesenchymal stromal cells isolated from human endometrium and endometriosis lesions. Bull Exp Biol Med 161: 610-615, 2016.

35. Savilova AM, Farkhat KN, Yushina MN, Rudimova YV, Makiyan ZN and Adamyan LV: Characteristics of multipotent mesenchymal stromal cells isolated from the endometrium and endometriosis lesions of women with malformations of the internal reproductive organs. Bull Exp Biol Med 162: 539-544, 2017.

36. Kao AP, Wang KH, Long CY, Chai CY, Tsai CF, Hsieh TH, Hsu CY, Chang CC, Lee JN and Tsai EM: Interleukin- $1 \beta$ induces cyclooxygenase- 2 expression and promotes the invasive ability of human mesenchymal stem cells derived from ovarian endometrioma. Fertil Steril 96: 678-684 e671, 2011.

37. Canosa S, Moggio A, Brossa A, Pittatore G, Marchino GL, Leoncini S, Benedetto C, Revelli A and Bussolati B: Angiogenic properties of endometrial mesenchymal stromal cells in endothelial co-culture: An in vitro model of endometriosis. Mol Hum Reprod 23: 187-198, 2017.

38. Hapangama DK, Drury J, Da Silva L, Al-Lamee H, Earp A, Valentijn AJ, Edirisinghe DP, Murray PA, Fazleabas AT and Gargett CE: Abnormally located SSEA1+/SOX9+ endometrial epithelial cells with a basalis-like phenotype in the eutopic functionalis layer may play a role in the pathogenesis of endometriosis. Hum Reprod 34: 56-68, 2019.

39. Esfandiari N, Nazemian Z and Casper RF: Three-dimensiona culture of endometrial cells: An in vitro model of endometriosis. Am J Reprod Immunol 60: 283-289, 2008.

40. Fasciani A, Bocci G, Xu J, Bielecki R, Greenblatt E, Leyland N and Casper RF: Three-dimensional in vitro culture of endometrial explants mimics the early stages of endometriosis. Fertil Steril 80: 1137-1143, 2003.

41. Prechapanich J, Kajihara T, Fujita K, Sato K, Uchino S, Tanaka K, Matsumoto S, Akita M, Nagashima M, Brosens JJ and Ishihara O: Effect of a dienogest for an experimental three-dimensional endometrial culture model for endometriosis. Med Mol Morphol 47: 189-195, 2014.

42. Esfandiari N, Ai J, Nazemian Z, Javed MH, Gotlieb L and Casper RF: Expression of glycodelin and cyclooxygenase-2 in human endometrial tissue following three-dimensional culture. Am J Reprod Immunol 57: 49-54, 2007. 
43. Esfandiari N, Khazaei M, Ai J, Bielecki R, Gotlieb L, Ryan E and Casper RF: Effect of a statin on an in vitro model of endometriosis. Fertil Steril 87: 257-262, 2007.

44. Maas JW, Le Noble FA, Dunselman GA, de Goeij AF, Struyker Boudier HA and Evers JL: The chick embryo chorioallantoic membrane as a model to investigate the angiogenic properties of human endometrium. Gynecol Obstet Invest 48: 108-112, 1999.

45. Nap AW, Groothuis PG, Demir AY, Maas JW, Dunselman GA, de Goeij AF and Evers JL: Tissue integrity is essential for ectopic implantation of human endometrium in the chicken chorioallantoic membrane. Hum Reprod 18: 30-34, 2003.

46. Nap AW, Groothuis PG, Punyadeera C, Klein-Hitpass L, Kamps R, Delvoux B and Dunselman GA: Oral contraceptives prevent the development of endometriosis in the chicken chorioallantoic membrane model. Contraception 78: 257-265, 2008.

47. Nap AW, Dunselman GA, Griffioen AW, Mayo KH, Evers JL and Groothuis PG: Angiostatic agents prevent the development of endometriosis-like lesions in the chicken chorioallantoic membrane. Fertil Steril 83: 793-795, 2005.

48. Koks CA, Groothuis PG, Dunselman GA, de Goeij AF and Evers JL: Adhesion of shed menstrual tissue in an in-vitro model using amnion and peritoneum: A light and electron microscopic study. Hum Reprod 14: 816-1822, 1999

49. van der Linden PJ, de Goeij AF, Dunselman GA, Erkens HW and Evers JL: Amniotic membrane as an in vitro model for endometrium-extracellular matrix interactions. Gynecol Obstet Invest 45: 7-11, 1998.

50. Groothuis PG, Koks CA, de Goeij AF, Dunselman GA, Arends JW and Evers JL: Adhesion of human endometrium to the epithelial lining and extracellular matrix of amnion in vitro: An electron microscopic study. Hum Reprod 13: 2275-2281, 1998.

51. van der Linden PJ, de Goeij AF, Dunselman GA, Erkens HW and Evers JL: Endometrial cell adhesion in an in vitro model using intact amniotic membranes. Fertil Steril 65: 76-80, 1996

52. Witz CA, Dechaud H, Montoya-Rodriguez IA, Thomas MR, Nair AS, Centonze VE and Schenken RS: An in vitro model to study the pathogenesis of the early endometriosis lesion. Ann N Y Acad Sci 955: 296-307, 340-342, 396-406, 2002.

53. Witz CA, Monotoya-Rodriguez IA and Schenken RS: Whole explants of peritoneum and endometrium: A novel model of the early endometriosis lesion. Fertil Steril 71: 56-60, 1999.

54. Groothuis PG, Koks CA, de Goeij AF, Dunselman GA, Arends JW and Evers JL: Adhesion of human endometrial fragments to peritoneum in vitro. Fertil Steril 71: 1119-1124, 1999.

55. Wild RA,Zhang RJ and Medders D: Whole endometrial fragments form characteristics of in vivo endometriosis in a mesothelial cel co-culture system: An in vitro model for the study of the histogenesis of endometriosis. J Soc Gynecol Investig 1: 65-68, 1994.

56. Lucidi RS, Witz CA, Chrisco M, Binkley PA, Shain SA and Schenken RS: A novel in vitro model of the early endometriotic lesion demonstrates that attachment of endometrial cells to mesothelial cells is dependent on the source of endometrial cells. Fertil Steril 84: 16-21, 2005.

57. Debrock S, De Strooper B, Vander Perre S, Hill JA and D'Hooghe TM: Tumour necrosis factor-alpha, interleukin-6 and interleukin-8 do not promote adhesion of human endometrial epithelial cells to mesothelial cells in a quantitative in vitro model. Hum Reprod 21: 605-609, 2006.
58. Griffith JS, Binkley PA, Kirma NB, Schenken RS, Witz CA and Tekmal RR: Imatinib decreases endometrial stromal cell transmesothial migration and proliferation in the extracellular matrix of modeled peritoneum. Fertil Steril 94: 2531-2535, 2010.

59. Nair AS, Nair HB, Lucidi RS, Kirchner AJ, Schenken RS, Tekmal RR and Witz CA: Modeling the early endometriotic lesion: Mesothelium-endometrial cell co-culture increases endometrial invasion and alters mesothelial and endometrial gene transcription. Fertil Steril 90: 1487-1495, 2008

60. Chen Z, Dai Y, Dong Z, Li M, Mu X, Zhang R, Wang Z, Zhang W, Lang J, Leng J and Jiang X: Co-cultured endometrial stromal cells and peritoneal mesothelial cells for an in vitro model of endometriosis. Integr Biol (Camb) 4: 1090-1095, 2012.

61. Yu J, Wang Y, Zhou WH, Wang L, He YY and Li DJ: Combination of estrogen and dioxin is involved in the pathogenesis of endometriosis by promoting chemokine secretion and invasion of endometrial stromal cells. Hum Reprod 23: 1614-1626, 2008.

62. Loh FH, Bongso A, Fong CY, Koh DR, Lee SH and Zhao HQ: Effects of peritoneal macrophages from women with endometriosis on endometrial cellular proliferation in an in vitro coculture model. Fertil Steril 72: 533-538, 1999

63. Takaoka O, Mori T, Ito F, Okimura H, Kataoka H, Tanaka Y, Koshiba A, Kusuki I, Shigehiro S, Amami T and Kitawaki J: Daidzein-rich isoflavone aglycones inhibit cell growth and inflammation in endometriosis. J Steroid Biochem Mol Biol 181: 125-132, 2018.

64. Wu D, Lu P, Mi X and Miao J: Exosomal miR-214 from endometrial stromal cells inhibits endometriosis fibrosis. Mol Hum Reprod 24: 357-365, 2018.

65. Lawrenson K, Lee N, Torres HA, Lee JM, Brueggmann D, Rao PN, Noushmehr H and Gayther SA: Src as a novel therapeutic target for endometriosis. Gynecol Oncol 135: 100-107, 2014.

66. Ohtake H, Katabuchi H, Matsuura $\mathrm{K}$ and Okamura $\mathrm{H}$ : A novel in vitro experimental model for ovarian endometriosis: The three-dimensional culture of human ovarian surface epithelial cells in collagen gels. Fertil Steril 71: 50-55, 1999.

67. Vigano P, Candiani M, Monno A, Giacomini E, Vercellini P and Somigliana E: Time to redefine endometriosis including its pro-fibrotic nature. Hum Reprod 33: 347-352, 2018.

68. Zhang Q, Duan J, Liu X and Guo SW: Platelets drive smooth muscle metaplasia and fibrogenesis in endometriosis through epithelial-mesenchymal transition and fibroblast-to-myofibroblast transdifferentiation. Mol Cell Endocrinol 428: 1-16, 2016. 